\title{
ОБЕЗЗАРАЖИВАНИЕ И ИСПОЛЬЗОВАНИЕ АНТРОПОГЕННЫХ ОТХОДОВ В РЕСУРСОСБЕРЕГАЮЩИХ СТРОИТЕЛЬНЫХ ТЕХНОЛОГИЯХ
}

\author{
Маковкин Владимир Иванович', \\ assorti2030@yandex.ru \\ Замятин Николай Владимирович', \\ zamnv@gmail.com
}

\author{
Смирнов Геннадий Васильевич', \\ smirnov@main.tusur.ru \\ 1 Томский государственный университет систем управления и радиоэлектроники, \\ Россия, 634050, г. Томск, пр. Ленина, 40.
}

Актуальность. Проблема охраны окружающей среды является одной из острейших проблем настоящего времени. Многостороннее замусоривание всех геосфер неутилизированными отходами привело к резкому ухудшению состояния экологических систем, к гибели некоторых уникальных природных комплексов, к сокращению и исчезновению популяций отдельных видов растений и животных, к опасности непредсказуемых необратимых последствий, к которым могут привести результаты техногенной деятельности человека. Поэтому утилизация техногенных отходов производств, а тем более использование их в ресурсосберегающих технологиях, безусловно, весьма актуальны.

Цель работы: показать возможность утилизации, контроля и использования отходов производств, в частности фторангидрита, в ресурсосберегающих строительных технологиях.

Методы: химическая нейтрализация отходов, дезинтеграция сырья и получение из него сыпучего клинкерного материала, способы его контроля и применения в разнообразных рецептурах в строительных технологиях; лазерное сканирование поверхности полученной сыпучей смеси; аппроксимация модели поверхности с применением радиальных базисных функций нейронных сетей; создание модели нелинейной функции поверхности по теореме Колмогорова с применением суперпозиции радиальных базисных функций; вычисление объема вещества, ограниченного полученной функцией с применением кубатурной функции методом Гаусса-Кронрода и методом Монте-Карло.

Результаты. Рассмотрена технология нейтрализации сульфаткальциевых отходов производства и технологическая схема его дезинтеграции и использования в буровых растворах. Представлена система контроля объема дезинтегрированного вещества, необходимая для учета его количества и дозирования при использовании в рецептурах различных технологий. Показано, что при контроле и оценке количества дезинтегрированного вещества лучшим является способ с аппроксимацией нелинейной функции, так как имеется возможность регулирования ошибки количеством разбиений функции или количеством опытов, а также учитывается модель поверхности вещества. Использование радиальных базисных функций нейронной сети целесообразно для получения модели поверхности сыпучих веществ с целью повышения точности измерения объемов в резервуарных парках и складах производственных предприятий. Наиболее эффективными являются методы моделирования функций поверхности и измерения их объема методами квадратур или Монте-Карло. Использование метода Гаусса-Кронрода в данном случае предпочтительно.

\section{Ключевые слова:}

Производственные отходы, ресурсосберегающие технологии, резервуар, уровнемер, объем, сыпучий материал, нейронные сети, сети радиальных базисных бункций, квадратурные функции, аппроксимирующая функция, интерполяционная функция, Matlab.

\section{Введение}

Одним из распространенных видов промышленных отходов химической промышленности являются сульфаткальциевые отходы, которые в значительной мере загрязняют окружающую среду.

В Обском бассейне России и Казахстана сульфаткальциевые отходы образуются на следующих промышленных предприятиях: ОАО «СХК», г. Томск; ОАО «Галополимер», г. Пермь; ОАО «Полевской криолитовый завод», г. Полевской, Свердловская обл.; АО «УМЗ», г. Усть-Каменогорск, Казахстан.

В процессе хозяйственной деятельности ОАО "СХК» образуется сульфаткальциевый отход фторангидрит - в количестве (тонн в год) 13500 , OАО «Галополимер» - 70000, ОАО «Полевской криолитовый завод» - 200000, АО «УМЗ»- 17000.
Фторангидрит образуется при получении фтороводорода в присутствии избытка серной кислоты, поэтому его необходимо нейтрализовать щелочным реагентом для перевода в твердое безопасное состояние. Для того чтобы полученный твердый материал не выбрасывать на свалку, загрязняя окружающую среду, а использовать его во благо людям, в частности, в ресурсосберегающих технологиях, его дезинтегрируют и хранят в специальных силосах. Обезвреженный по сухому способу фторангидрит, а также техногенный ангидрит, модифицированный солями одновалентных металлов, обладают весьма востребованными в строительной промышленности качествами пластификатора, ангидритового вяжущего, пигмента, наполнителя. Например, фторангидрит подходит для изготовления сухих строительных смесей, 
гипсоволокнистых листов (ГВЛ), гипсокартонных листов (ГКЛ), гипсовых плит и блоков, и пр. [1-6].

Для хранения и использования материалов на предприятиях используется совокупность различных резервуаров, называемых резервуарным парком, который должен эксплуатироваться максимально эффективно при минимальных затратах. Актуальной задачей в данной области является создание технологий по преобразованию и применению техногенных отходов, в частности фторангидрита, и быстрое получение точной информации об имеющихся на предприятии объемах этих веществ, а также о динамике их применения [7].

\section{Технологическая схема нейтрализации}

\section{и дезинтеграции фторангидрита}

Учеными Томского политехнического университета был разработан и внедрен в производство уникальный способ возведения помещений различного назначения (гаражные боксы, склады, киоски и пр.). Производство состоит из следую- щих этапов: изготовление металлического каркаса, обнесение каркаса съемной опалубкой, заливка межопалубочного пространства строительной смесью из техногенных отходов: фторангидрита и золошлака. Золошлак выступает в качестве инертного материала. Из фторангидрита $\mathrm{CaSO}_{4}$ получают ангидритовое вяжущее [8]. Ангидритовое вяжущее - это унифицированный ангидрит (нейтрализованный, усредненный по массе, классифицированный по фракциям и модифицированный сульфатом 1-го валентного металла, предпочтительно $\mathrm{K}_{2} \mathrm{SO}_{4}$ в количестве $1,5 \%$ от общей массы фторангидрита). После кристаллизации (схватывания смеси) двуводного гипса $\mathrm{CaSO}_{4} \cdot 2 \mathrm{H}_{2} \mathrm{O}$ и снятия опалубки готовые модули транспортируются и монтируются по месту строительства [9-11].

Кроме производства каркасно-монолитных помещений выпускается унифицированный техногенный ангидрит и ангидритовое вяжущее; листы «ПАНО» (Панелей Ангидритовых Отделочных); профильные строительные изделия типа «швел-

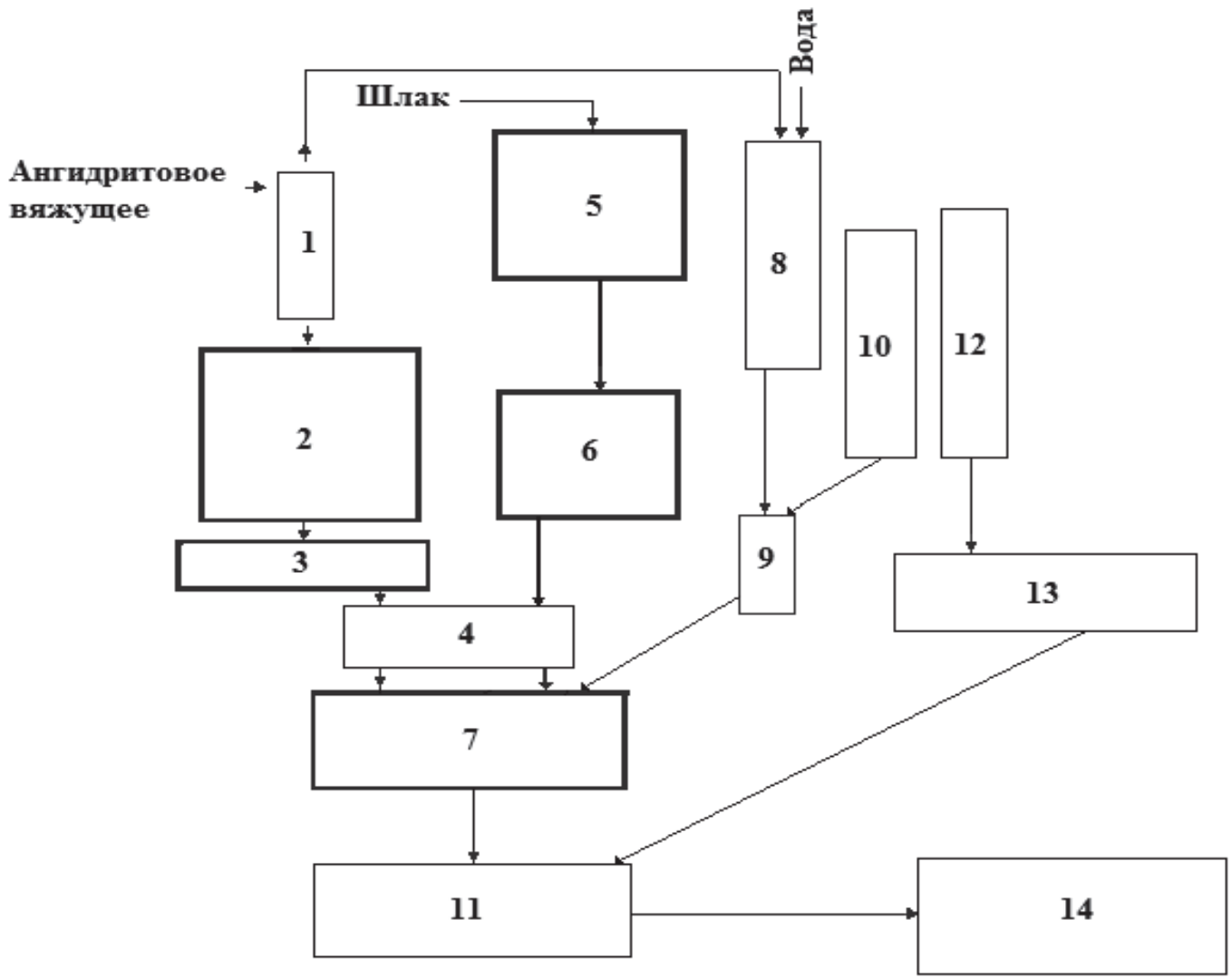

Рис. 1. Технологическая схема изготовления ангидритовых каркасно-монолитных модулей: 1 - циклон; 2 - расходный бункер ангидритового вяжущего; 3 - шнек-дозатор ангидритового вяжущего; 4 - скип; 5 - расходный бункер золошлака; 6 шнек-дозатор золошлака; 7 - растворо-бетоно-смеситель; 8 - абсорбер; 9 - дозатор воды; 10 - расходная емкость с водой; 11 - площадка изготовления каркасно-монолитных модулей; 12 - склад хранения металлопроката и съемной опалубки; 13 - площадка изготовления металлического каркаса модуля; 14 - склад полуфабрикатной продукции - каркасно-монолитных модулей помещений

Fig. 1. Technological scheme for manufacturing anhydrite frame-monolithic modules: 1 is the cyclone; 2 is the consumption bunker of anhydrite binder; 3 is the auger screw of anhydrite binder; 4 is the skip; 5 is the consumption bunker of ash-and-slag; 6 is the auger screw of ash-and-slag; 7 is the concrete mixer for mortar; 8 is the absorber; 9 is the water batcher; 10 is the tank with water; 11 is the area for manufacturing frame-monolithic modules; 12 is the storage warehouse for rolled metal products and removable formwork; 13 is the area for manufacturing metal framework of the module; 14 is the warehouse of semi-finished products - frame-monolithic modules of buildings 
лер», используемые при возведении несъемной опалубки, ограждений территорий, межкомнатных перегородок и пр. Наиболее экономически эффективной из вышеперечисленных продукций являются листы «ПАНО», по своим характеристикам превосходящие гипсокартонные и гипсоволокнистые листы фирмы «Knauf» .

Процесс нейтрализации фторангидрита осуществляется гидроксидом кальция $\mathrm{Ca}(\mathrm{OH})_{2}$ по реакции 1:

$$
\mathrm{H}_{2} \mathrm{SO}_{4}+\mathrm{Ca}(\mathrm{OH})_{2}=\mathrm{CaSO}_{4}+2 \mathrm{H}_{2} \mathrm{O} .
$$

Технологическая схема изготовления ангидритовых каркасно-монолитных модулей показана на рис. 1.

Первоначально из цеха подготовки унифицированного фтторангидрита по пневмопроводу через циклон - 1 подается ангидритовое вяжущее $(\mathrm{AB})$ в расходный бункер - 2 ангидритового вяжущего. Из бункера - 2 посредством шнека-дозатора - 3 ангидритовое вяжущее поступает в скип - 4. Просеянный золошлак (фракцией менее 20 мм) разгружают грузовым транспортом в расходный бункер 5 и при помощи шнека-дозатора - 6 подают также в скип - 4, из которого сыпучие материалы поступают в растворо-бетоносмеситель (РБС) - 7 . В РБС - 7 из емкости - 10 через дозатор - 9 подается вода. Запыленный воздух после циклона - 1 поступает в водяной абсорбер - 8, что обеспечивает безотходность производства. После завершения наполнения бункера - 2 циркуляция воды через абсорбер - 8 прекращается и пульпа (взвесь ангидрита в воде) направляется через дозатор - 9 в РБС - 7. После смешения исходных ингредиентов полученный ангидритошлаковый бетон заливают в межопалубочное пространство многократно используемой съемной опалубки предварительного смонтированного вокруг металлического каркаса на площадке изготовления модуля - 11. Каркас модуля изготавливают на площадке производства металлического каркаса модуля - 13 из металлопроката, заранее привезенного на склад - 12 .

Актуальной проблемой на всех этапах производства является определение объема вещества и получаемых смесей в бункерах (или, возможно, других резервуарах).

\section{Актуальность проблемы}

Для определения объема веществ на практике применяют несколько способов. Самый простой из них - это определение объема вручную простукиванием или буйковым методом. Однако чаще используют цифровые и аналоговые уровнемеры [12-14]. Если вещество сыпучее, вязкое или имеет другую консистенцию, отличную от жидкой, то оно образует неровную поверхность, выпуклой или вогнутой формы (в зависимости от того, наполняется резервуар или из него откачивается смесь). Искривленная поверхность материала может возникать в результате его сил сцепления с поверхностью резервуара, что приводит к его налипанию к стенкам резервуара (рис. 2). В подобной ситуации объем смеси в резервуаре вычисляется по усредненному или другому доступному (обычно уровнемер находится в фиксированном положении и может измерять значения только в определенном месте) значению уровня. Такое определение объема имеет большие погрешности, нарастающие с каждым контролируемым резервуаром.


Рис. 2. Форма поверхности при наполнении или откачке вещества в резервуаре: 1 - уровнемер; 2 - поверхность вещества: черное - реальная поверхность, красное предполагаемая поверхность, под которой обычно осуществляется измерение объема

Fig. 2. Shape of surface while filling or pumping out the substance in the silo: 1 is the level gauge; 2 is the surface of substance: black - real surface, red - the assumed surface under which the volume measurement is usually carried out

Из-за недостаточной точности определения объемов веществ в резервуарах на предприятиях возникают следующие проблемы:

- низкая скорость сбора, передачи и анализа данных о количестве веществ на резервуарных парках, которая особенно актуальна, если парки распределены на всем предприятии или находятся в разных регионах;

- низкая точность получаемых данных о количестве веществ на резервуарных парках;

- простои из-за неверных расчетов динамики использования или производства сырья;

- низкое качество получаемых смесей из-за погрешностей при расчете процентного соотношения веществ в различной рецептуре;

- дополнительные затраты из-за сопутствующих ошибок в бухгалтерии, неверных расчетов объемов производства, динамики использования сырья и т. д.;

- низкая культура труда в резервуарных парках. Объем веществ в резервуарах может вычисляться разными способами [15], такими как:

- визуальный метод - замеры на глаз, простукивание резервуара, использование буйков, лине- 
ек и тому подобных вспомогательных устройств. Наименее точный метод, погрешность которого может составлять до $30 \%$;

- теоретический расчет - расчет разницы количества поступающего и вывозимого материала. Подобные измерения редко применяют на практике, так как их невозможно использовать при производстве смесей и любых других операциях, требующих точных значений объемов;

- маркшейдерский залер - использование специализированных знаний, свойств сыпучих материалов, реологических свойств веществ и значений высоты вершины горки и ее диаметра. На основе полученных данных прорабатывается формула и по ней рассчитывается объем;

- лазерное сканирование поверхности - использование системы уровнемеров или подвижных уровнемеров для измерения высот в различных точках, моделирование поверхности в виде нелинейной функции и затем вычисление объема, ограниченного этой поверхностью.

Выбор определенного метода измерения объема базируется на разных факторах и зависит от требуемой точности. Потенциально, меньшую погрешность имеет метод построения трехмерных поверхностей веществ со значениями уровня в каждой точке.

\section{Постановка задачи}

С целью повышения точности определения объемов сыпучих веществ в резервуарах необходимо проанализировать способы определения объемов под нелинейной поверхностью, обосновать наиболее вероятную аппроксимацию функции поверхности, исследовать возможности применения аппроксимации посредством нейронных сетей и криволинейных интегралов, выполнить экспериментальную проверку. Для выполнения поставленной задачи предложена следующая последовательность действий:

1) получение дискретного количества координат уровней поверхности вещества;

2) аппроксимация поверхности модели для получения промежуточных неизвестных значений координат и ее нелинейной функции;

3) вычисление объема вещества под образуемой поверхностью.

\section{Аппроксимация модели поверхности сыпучего вещества}

Чаще всего для моделирования поверхности по известным координатам применяют математические модели построения рельефа, в частности аппроксимацию высот между смежными точками модели. Тогда задача определения уровня на поверхности координат решается нахождением в каждом случае трех смежных исходных точек модели, между которыми должна находиться соответствующая искомая точка, определением коэффициентов уравнения плоскости, проходящей через эти три точки, вычислением по полученному уравнению высоты [16].
Более универсальными являются статистические модели - «плавающего квадрата» и «плавающего круга», которые описаны в работе [17].

Для математического моделирования поверхностей предложено использовать нейронные сети, обладающие высокой скоростью и точностью получаемых моделей.

В работах $[16,18]$ проведены сравнения различных нейронных сетей, в том числе и для аппроксимации входных значений. Сравнение точностей выходов нейронных сетей в работе [18] проводилось по коэффициентам детерминации, представленным в табл. 1.

Таблица 1. Качество аппроксимации по коэффициенту детерминации

Table 1. Quality of approximation by the determination coefficient

\begin{tabular}{|c|c|c|c|}
\hline $\begin{array}{c}\text { Наименование } \\
\text { Title }\end{array}$ & $\begin{array}{c}\text { GRNN сеть } \\
\text { GRNN network }\end{array}$ & $\begin{array}{c}\text { РБФ сеть } \\
\text { RBF network }\end{array}$ & $\begin{array}{c}\text { Линейная сеть } \\
\text { Linear neural network }\end{array}$ \\
\hline $\begin{array}{c}\text { Коэффициент } \\
\text { Coefficient }\left(R^{2}\right)\end{array}$ & 0,9473 & 0,9918 & 0,6422 \\
\hline
\end{tabular}

Другие модели, используемые для сглаживания функции по точкам, такие как ряды Фурье и мультипликативные модели, имеют следующие коэффициенты детерминации:

- ряды Фурье: 0,8747;

- мультипликативные модели: 0,7262.

Из приведенных данных видно, что лучше всего для аппроксимации поверхностей использовать РБФ и GRNN нейронные сети. При использовании РБФ сети получен коэффициент детерминации $R^{2}=0,9918$. Это означает, что реальный выход нейросети и желаемый выход (что по смыслу совпадает с оценочными и реальными значениями) практически совпадают. Применяя аналитические или численные методы, практически невозможно достичь такого высокого значения коэффициента детерминации. В плане скорости выполнения операций и обучения сети для аппроксимации модели поверхности лучше подходят РБФ сети, так как они не имеют второго промежуточного слоя, отвечающего в GRNN за оценку взвешенного среднего.

Таким образом, для аппроксимации данных по известным координатам уровня целесообразно применение нейронных сетей, в частности РБФ сети, которая обеспечивает высокое качество аппроксимации и может использоваться для моделирования поверхности веществ.

Рассмотрим способ применения нейронных сетей для моделирования поверхности сыпучих веществ.

В 1956 г. Колмогоровым было доказано, что любая непрерывная функция может быть реализована в виде суперпозиции функций меньшей размерности [19]. Неявная функция трехмерной поверхности может быть представлена в виде (2):

$$
f(x, y)=\sum_{i=1}^{n} \omega_{i} \varphi(r)
$$


где $\omega_{i}$ - константы; $\varphi(r)$ - радиально-базисная функция.

В качестве функции $\varphi(r)$ можно выбрать любые радиально-базисные функции (3), которые широко используются для решения задач многомерной интерполяции и аппроксимации

$$
\varphi\left(x, y, c_{i}^{x}, c_{i}^{y}\right)=1-\sqrt{\left(x-c_{i}^{x}\right)^{2}+\left(y-c_{i}^{y}\right)^{2}},
$$

где $c_{i}^{x}, c_{i}^{y}$ - некоторые константы, называемые ядрами РБФ.

Неявная функция поверхности может быть реализована с помощью нейронной сети, состоящей из двух слоев: скрытого нелинейного слоя, имеющего n нейронов и выходного линейного слоя, имеющего один нейрон, который передает на выход взвешенную сумму выходов нейронов первого слоя. Структура такой нейронной сети представлена на рис. 3.

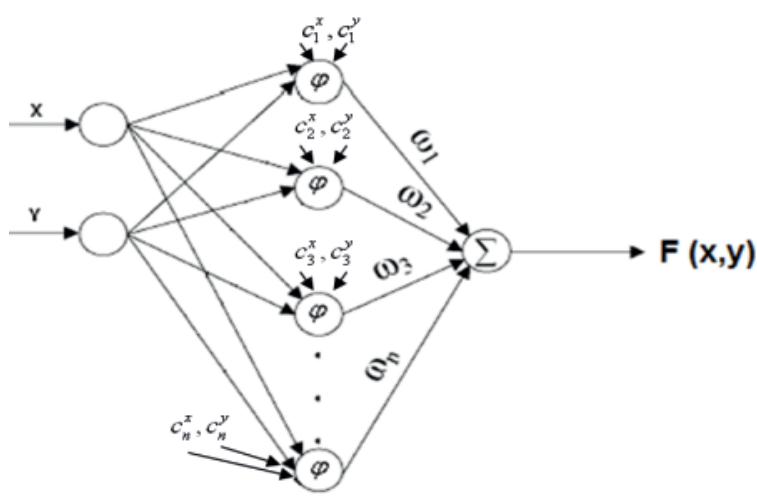

Рис. 3. Нейронная сеть РБФ: $x, y$-координаты точек поверхности вещества; $c_{i}^{x}, c_{i}^{y}-$ ядра РБФ; $\varphi$ - активационная радиальная базисная функция; $\omega_{i}-$ веса синапсов; $F(x, y)$ - функция зависимости уровня от входных ко ординат $x, y$

Fig. 3. RBF neural network: $x, y$ are the coordinates of substance surface points $C_{i}^{x}, C_{i}^{y}$ are the RBF cores; $\varphi$ is the activation radial basis function; $\omega_{i}$ are the synaptic weights; $F(x, y)$ is the function of level dependence on input coordinates $x, y$

Алгоритм обучения нейронной сети с использованием РБФ выглядит следующим образом:

- выбирают число нейронов в скрытом слое, равное количеству входных точек;

- для каждого нейрона задают ядро РБФ $\left(c_{i}^{x}, c_{i}^{y}\right)$ таким образом, что $c_{i}^{x}=x_{i}, c_{i}^{y}=y_{i}$;

- определяют веса $\omega_{i}$.

Выходное значение сети для $i$-х входных данных определяется по формуле (4)

$$
\begin{gathered}
D_{i}=w_{1} f\left(x_{i}, y_{i}, c_{1}^{x}, c_{1}^{y}\right)+w_{2} f\left(x_{i}, y_{i}, c_{2}^{x}, c_{2}^{y}\right)+\ldots+ \\
+w_{n} f\left(x_{i}, y_{i}, c_{n}^{x}, c_{n}^{y}\right) .
\end{gathered}
$$

Таким образом, рельеф поверхности представлен в виде нелинейной функции - суперпозиции РБФ обученной нейронной сети. Затем объем рассматриваемого вещества можно определить, peшив двойной или тройной интеграл уже известной функции.

\section{Способы измерения объема}

Погрешность вычислений объема зависит только от неровностей поверхности, так как объем смесей ниже минимального уровня поверхности смеси всегда одинаковый и определяется формой и размерами резервуара, независимо от значений других уровней. Объем вещества может быть измерен следующими способами.

Излерение объела по гладкой поверхности осуществляют по уровню в определенном месте (рис. 4, А). В этом случае для правильного определения объема необходима информация о характеристиках резервуара и уровня материала или сырья, получаемого с помощью уровнемера. Предполагается, что поверхность смеси гладкая и уровень смеси в любом месте остается неизменным, как если бы измерялся объем жидкости [20, 21].
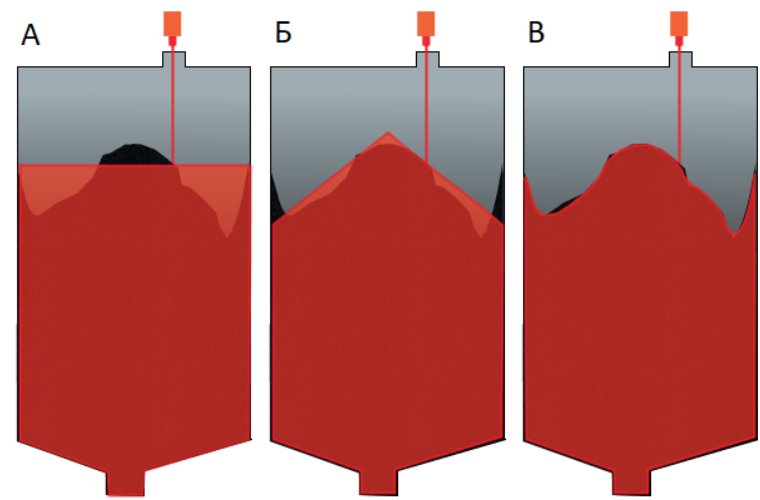

Рис. 4. Графическое представление способа измерения объема вещества (A) по гладкой поверхности, (Б) по конусу, (B) по нелинейной функции поверхности

Fig. 4. Graphical representation of the method for measuring the volume of the substance (A) by a smooth surface, $(\bar{B})$ by the cone, $(B)$ by the nonlinear function of the surface

Объем вычисляется по формуле объема цилиндра (5), если используется вертикальный цилиндрический резервуар:

$$
V=\pi r^{2} h,
$$

где $r$ - радиус резервуара; $h$ - уровень смеси.

При использовании резервуаров иных видов необходимо выбрать другую формулу, например, объем усеченного цилиндра, если смесь хранится в горизонтальном резервуаре цилиндрической формы. Данный способ остается самым простым, чаще всего именно его используют при измерениях вручную, так как он является самым быстрым из рассмотренных и не требует сложных расчетов. Но, применяя его, игнорируются реологические и сыпучие свойства смесей, и точность подобного способа самая низкая из предложенных выше.

Излерение объела по конусу - вычислить объем по уровню вещества в определенном месте и моделируемому конусу или конусовидному углублению (при наполнении и откачке соответственно) с углом между образующими и основанием ко- 
нуса, равным углу естественного откоса соответствующего материала (рис. 4, Б). Необходимы характеристики резервуара, координаты положения лазерного уровнемера и получаемые им значения уровня, угол естественного откоса используемого материала или смеси. Угол естественного откоса (иногда также угол внутреннего трения, угол ската) - угол, образованный свободной поверхностью рыхлой горной массы или иного сыпучего материала с горизонтальной плоскостью (рис. 5).

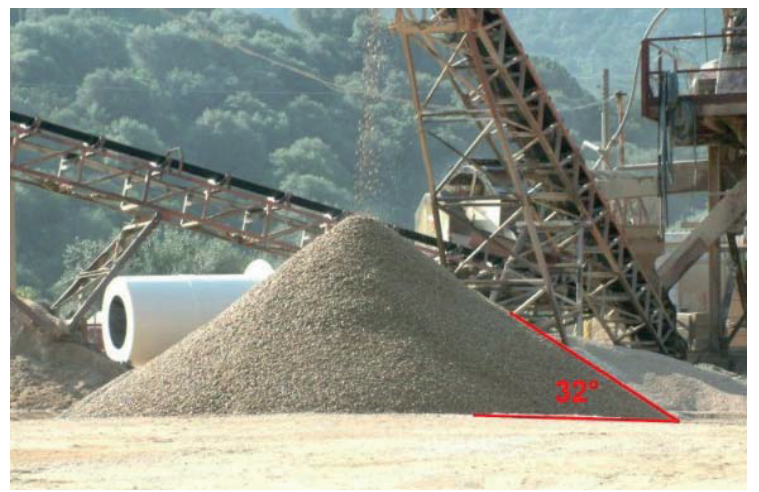

Рис. 5. Форма поверхности и угол естественного откоса гравия

Fig. 5. Shape of surface and gravel repose angle

Кроме этого, достаточно знать место наполнения/откачки смеси, которое может совпадать с вершиной конуса, и характеристики корпуса резервуара, ограничивающие конус. Для примера объем образуемой материалом «горки» в вертикальном цилиндрическом резервуаре можно рассчитать с помощью формулы (6):

$$
v=\frac{1}{3} \pi r^{2} h_{\text {осн }},
$$

где $r$ - радиус резервуара; $h_{\text {осн }}$ - высота «горки» сыпучего материала.

Положение уровнемера может быть ограничено из-за конструкции резервуара, например, он может быть помещен только в отверстии выхода воздуха, так как резервуар имеет закрытую форму и нет возможности его установки непосредственно над вершиной конуса. Но, определив угол естественного откоса и характеристики резервуара, можно определить высоту «горки», пользуясь правилом прямоугольного треугольника, и получить формулу (7):

$$
h_{\text {осн }}=r \operatorname{tg} \alpha \text {, }
$$

где $r$ - радиус резервуара; $\alpha$ - угол естественного откоса сыпучего материала.

Использование угла естественного откоса и вычисления объема конуса значительно увеличивает точность по сравнению с измерением объема по гладкой поверхности, но имеются следующие недостатки:

- требуется больше данных и предварительные математические расчеты перед эксплуатацией на различных типах резервуаров;

- угол естественного откоса варьируется в зависимости от влажности, типа сыпучего материа- ла и времени (возможны оседание материала, уменьшение пористости, влияние влажности во времени и т. д.) и требует предварительных вычислений с помощью специальных приборов;

- угол между основанием и рыхлой массой равен углу естественного откоса только при наполнении или откачке из резервуара, но между этими состояниями при переходах от первого процесса ко второму и наоборот, угол также будет меняться.

Излерение объела по нелинейной функции поверхности - вычисление объема по дискретному количеству координат уровней и по моделируемой нелинейной функции, описывающей поверхность сыпучего материала с определенной точностью (рис. 4, В). Необходимы характеристики резервуара и модель поверхности в виде ее нелинейной функции.

Объем вещества можно найти, используя двойной интеграл известной функции поверхности, ограниченной на заданном пространстве, или применить метод Монте-Карло.

Определить объем вещества в вертикальном цилиндрическом резервуаре можно, исходя из геометрического смысла двойного интеграла. Если функция $f(x, y)$ принимает в области $D$ только положительные значения, двойной интеграл данной функции численно равен объему $V$ вертикального цилиндрического тела, построенного на основании $D$ и ограниченного сверху соответствующим куском поверхности $z=f(x, y)(8)$.

$$
V=\iint_{D} z d x d y .
$$

Вычисление значений интегралов возможно двумя распространенными способами:

- использованием квадратурных или кубатурных формул;

- использованием метода Монте-Карло.

\section{Экспериментальные результаты}

Возможность использования предложенной методики проверялась экспериментально на макете вертикального цилиндрического резервуара, и в качестве измеряемого материала использовался цемент M400. Для измерения значений уровня использовалась цилиндрическая система координат с осью $0 z$, проходящей через центр модели. В реальных условиях на вход РБФ нейронной сети можно подавать неравномерно распределенные данные, но для упрощения исследовательской работы точки расположены на равных расстояниях друг от друга, значения уровня измерялись на одинаковых интервалах $\varphi$ и $r$ друг от друга.

В качестве измерителя уровня использовался лазерный дальномер SICK DT50 (погрешность \pm 1 мм). Диаметр резервуара: 23,28 см. Шаг по $r$ : 3,88 и 1,94. Шаг по $\varphi: 30$ и 15 градусов. Фрагмент полученных данных представлен в табл. 2 .

Графический пример полученных данных представлен на рис. 6. 
Таблица 2. Координаты уровней, полученные с поверхности цемента

Table 2. Coordinates of levels obtained from the surface of cement

\begin{tabular}{|c|c|c|c|c|}
\hline$\varphi$, град $\backslash r, \mathrm{~cm} / \varphi$, deg $\backslash r, \mathrm{~cm}$ & 0 & 3,9 & 7,8 & 11,7 \\
\hline 0 & 9 & 4,7 & 1,3 & 0 \\
\hline 30 & 9 & 5,4 & 3,4 & 2,3 \\
\hline 60 & 8,6 & 6,9 & 4,1 & 3,3 \\
\hline 90 & 8,5 & 7,1 & 4,1 & 3,3 \\
\hline 120 & 8,6 & 7 & 4,7 & 3,3 \\
\hline 150 & 8,6 & 6 & 4 & 3,4 \\
\hline 180 & 9 & 7 & 4,3 & 2 \\
\hline 210 & 9 & 6,3 & 3,8 & 2 \\
\hline 240 & 8,6 & 6,9 & 3,6 & 1,3 \\
\hline 270 & 8,5 & 6,9 & 3,3 & 0,4 \\
\hline 300 & 8,6 & 6,3 & 2,8 & 0,5 \\
\hline 330 & 8,6 & 6,6 & 2,4 & 0,7 \\
\hline
\end{tabular}

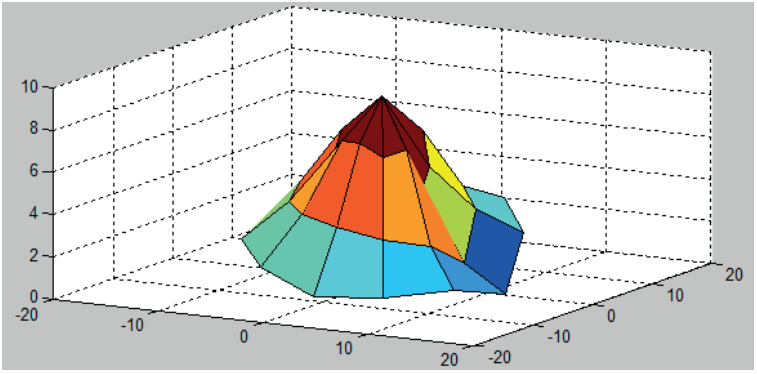

Рис. 6. Графическое представление полученных данных

Fig. 6. Graphical representation of the data obtained

Так как применяемая радиально базисная функция не работает с цилиндрическими координатами напрямую из-за евклидова расстояния между центром и точками, проведена замена переменных по закону преобразования координат от цилиндрических к декартовым по формуле (9):

$$
\left\{\begin{array}{l}
x=r \cos \varphi ; \\
y=r \sin \varphi,
\end{array}\right.
$$

где $r$ - полярный радиус; $\varphi$ - полярный угол.

В качестве активационной функции нейронов используется кубическая РБФ функция (10). Выбор активационной функции подбирался экспериментально при сравнении с реальной поверхностью по внешнему виду полученной модели.

$$
a=n^{3},
$$

где $n$ - евклидово расстояние от ядра РБФ до координаты входной точки.

При обучении РБФ нейронной сети с нулевой ошибкой количество нейронов скрытого слоя всегда равно числу элементов обучаемого множества (значений, подаваемых на вход сети). С такой ошибкой нельзя получить приемлемого по скорости решения в случае больших размеров обучающего множества и необходимы дополнительные манипуляции с определением оптимальной погрешности получаемой модели. Также нулевая ошибка не дает гарантии, что промежуточные значения, на которых не была обучена нейронная сеть, дадут точный результат, а чаще наоборот, модель поверхности будет иметь сложную форму, отличающуюся от реальной.

При обучении нейронной сети с погрешностью менее одного процента для аппроксимации поверхности в данном эксперименте оказалось достаточно двенадцати нейронов. При обеспечении точности порядка 99 процентов этого достаточно, при этом значительно снизилось время расчетов. Пример работы нейронной сети с кубической активационной функцией изображен на рис. 7. Скорость и точность обучения сети изображена на рис. 8.

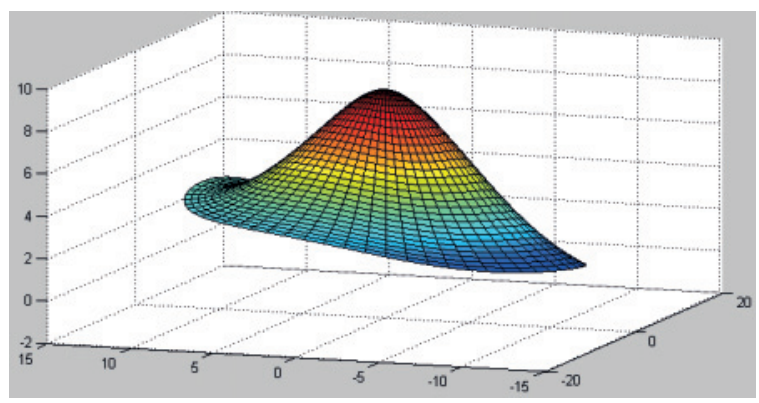

Рис. 7. Пример использования кубической активационной функции в нейронной сети

Fig. 7. Use of cubic activation function in a neural network

При оптимизации процесса обучения нейронной сети время обработки данных составляет десятые доли секунды и этого достаточно при использовании этого способа на резервуарных парках.

В статье исследуется поверхность в статическом состоянии (не учитывая влияние засыпания или откачки в динамике). Внимание уделяется измерению объема «горки», образованной веществом (объем смеси под ней остается неизменным) и значения уровня оцениваются от ее нижней границы. Объем смеси под горкой $V_{\text {осн }}$ рассчитывается по формуле объема вертикального цилиндра.

Измерение объема по гладкой поверхности осуществлялось по среднему уровню вещества с гладкой поверхностью, угол естественного откоса равен $40-50^{\circ}$. Для проверки достоверности данных значений оценены углы поверхности песка по касательной в каждой точке, из-за неровностей и комковатости поверхности средний угол получился равным $39^{\circ}$. Проверка показала, что полученные значения соответствуют табличным и в качестве угла естественного откоса выбран угол в $40^{\circ}$. В способе измерения объема по нелинейной функции двойной интеграл вычислялся методом Гаусса-Кронрода. Оценка погрешностей способов представлена в табл. 3.

Таблица 3. Погрешность способов измерения объема

Table 3. Error of ways of measuring the volume

\begin{tabular}{|c|c|c|}
\hline $\begin{array}{c}\text { Гладкая поверхность } \\
\text { Flat surface }\end{array}$ & $\begin{array}{c}\text { Конус } \\
\text { Cone }\end{array}$ & $\begin{array}{c}\text { Нелинейная функция } \\
\text { Nonlinear function }\end{array}$ \\
\hline \multicolumn{3}{|c|}{$\%$} \\
\hline 25,8 & 21,7 & 8 \\
\hline
\end{tabular}




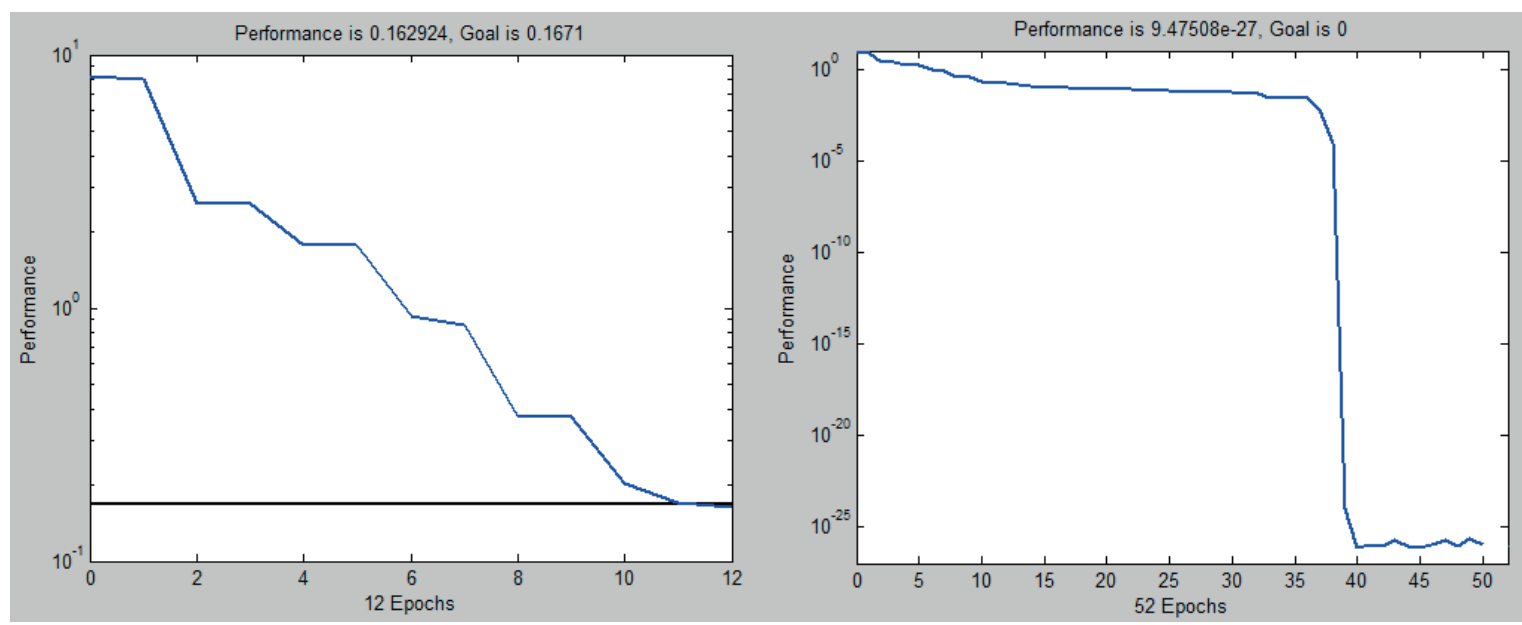

Рис. 8. Графики обучения РБФ нейронной сети с ошибкой 0,16 (слева) и нулевой ошибкой (справа)

Fig. 8. Graphs of training RBF neural networks with an error of 0,16 (left) and zero error (right)

\section{Заключение}

По результатам экспериментов выяснено, что объем веществ можно вычислить каждым из этих способов. Наибольшую ошибку имеет способ измерения объема по гладкой поверхности и чуть меньшую - по конусу. Проведя более подробный анализ характеристик смесей и разработав соответствующие формулы, можно увеличить точность измерений по конусу, но останутся его главные недостатки - узкая направленность на исследуемый тип смеси и трудоемкость исследовательской части.

Исходя из данных в табл. 3 и аналитически, из имеющихся трех способов лучшим является способ с аппроксимацией нелинейной функции, так как имеется возможность регулирования ошибки количеством разбиений функции или количеством опытов, а также учитывается модель поверхности вещества.

Использование РБФ нейронной сети целесообразно для получения модели поверхности сыпучих веществ с целью повышения точности измерения

\section{СПИСОК ЛИТЕРАТУРЫ}

1. Fedorchuk Yu.M., Zykova N.S., Mikheev V.N. The multipurpose use of industrial wastes in the process of producing anhydrite construction materials // Strategic Technology (IFOST), $20127^{\text {th }}$ 7th International Forum on Strategic Technology (IFOST). Tomsk, Russia, 2012. $-3 \mathrm{p}$.

2. Research of Gypsum-Containing Raw Materials for Obtaining of Dry Building Mixes / L. Anikanova, 0. Volkova, I. Guseva, A. Kurmangalieva // Key Engineering Materials. - 2015. V. $683 .-$ P. $295-300$

3. Gutt W. Manufacture of cement from industrial byproducts // Chemistry and Industry. - 1971. - № 7. - P. 189-197.

4. Garkavi M.S. Anhydrite Floors for Civil Construction // Proc. of the $14^{\text {th }}$ Internationale Baustofftagung «Ibausil». - Weimar, Germany, 2000. - P. 865-870.

5. Wang Jinhua, Yang Xinya, Yu Degao. Modification of fluorgypsum as cement retarder // Journal of Wuhan University of Technology-Mater. - 2007. - V. 22. - № 4. - P. 745-748.

6. Ludvigsen K. Production of gypsum boards from gypsum waste // Cim., betons, platres, chaux. - 1998. - № 2. - P. 97-98. объемов в резервуарных парках и складах производственных предприятий. Посредством РБФ сетей возможно аппроксимировать любые поверхности, если известны координаты их точек, вне зависимости от интервалов между ними. Можно также увеличить точность вычисления, рассмотрев точки, лучше всего характеризующие поверхность (точки в местах перехода поверхности от понижения к повышению уровня, точки с максимальными и минимальными уровнями и так далее). По этим причинам данный способ лучше подходит для точного измерения объема.

Наиболее эффективными являются методы моделирования функций поверхности и измерения их объема методами квадратур или Монте-Карло, так как они имеют наименьшую погрешность, исходя из полученных результатов. Использование метода Гаусса-Кронрода в данном случае предпочтительно, так как при разнице числа операций в тысячи раз его точность остается в несколько раз больше.

7. Маковкин В.И., Замятин Н.В. Система мониторинга и обработки информации о состоянии резервуарного парка // Доклады Томского государственного университета систем управления и радиоэлектроники. - 2016. - Т. 19. - № 4. - С. 95-99.

8. Prediction of the properties anhydrite construction mixtures based on neural network approach / Y.M. Fedorchuk, N.V. Zamyatin, G.V. Smirnov, 0.N. Rusina, M.A. Sadenova // Journal of Physics: Conference Series. - 2017. - № 881. -6 p.

9. Федорчук Ю.М. Результаты пуско-наладочных и технологических испытаний производства унификации ангидрита, получаемого из твердых отходов фтороводородного производства Сибирского химического комбината // Химическая промышленность. - 2004. - № 3. - С. 113-115.

10. Малогабаритное здание из бетонной смеси: пат. Рос. Федерация № 35540; заявл. 25.09.2003; опубл. 20.01.2004, Бюл. № $2 .-6$ с.

11. Способ контроля параметров сыпучих материалов в резервуарах: пат. Рос. Федерация № 2661314; заявл.16.05.2017; опубл. 13.07.2018, Бюл. № 20. - 16 с.

12. Намазбаев Т.С., Есенбаев С.Х., Колесников В.А. Средства измерения и контроля технологических процессов на предприя- 
тиях горно-металлургического комплекса. - Караганда: Изд-во КарГТУ, 2012. - 107 с.

13. Якимович Е.А., Замятин Н.В. Моделирование бесконтактного видеоуровнемера с использованием анализа нейронных сетей // Научное издание «Информационные технологии в территориальном управлении, промышленности, образовании»: Сб. статей. - Томск, 2002. - С. 165-170.

14. Смирнов Г.В., Смирнов Д.Г. Пропитка обмоток электрических машин магнитодиәлектрическим композитом с ультрадисперсным никель-цинковым наполнителем // Доклады Томского государственного университета систем управления и радиоэлектроники. - 2016. - Т. 19. - № 2. - С. 99-102.

15. Методы измерения объема сыпучих материалов / А.Г. Рыбаков, Е.В. Лукьянова, И.С. Бурмистрова, Ю.Ф. Муратова, М.В. Фёдорова // Энергетические и электротехнические системы: международный сборник научных трудов. - Магнитогорск: Магнитогорский государственный технический университет им. Г.И. Носова, 2015. - С. 430-433.

16. Кулажский А.В. Применение нейронных сетей на основе радиальных базисных функций в моделировании рельефа местности // Вестник РГУПС, Железнодорожный путь и транспортное строительство. - 2009. - Т. 35. - № 3. - С. 95-100.
17. Обоснование математической модели местности для проектирования автомобильных дорог / Н.Е. Коновалов, Н.Н. Зоннә, И.Г. Мельник, А.К. Якимович // Науч. Тр. Гипродорнии. 1976. - Вып. 18. - 7 с.

18. Филатова Т.В. Применение нейронных сетей для аппроксимации данных. - Томск: Изд-во Томский гос. университет, 2004. -30 c.

19. Колмогоров А.Н. 0 представлении непрерывных функций нескольких переменных суперпозициями непрерывных функций меньшего числа переменных // Доклады АН СССР. - 1956. T. 108. - C. 179-182.

20. Simultaneous detection of liquid level and refractive index with a long-period fiber grating based sensor device / Y. Huang, B. Chen, G. Chen, H. Xiao, S.U. Khan // Measurement Science and Technology. - 2013. - V. 24 (9). - 095303.

21. Khaliq S., James S.W., Tatam R.P. Fiber-optic liquid-level sensor using a long-period grating // Opt. Lett. - 2001. - V. 26. P. 1224-1226.

Поступила 15.02.2018 2.

\section{Информация об авторах}

Маковкин В.И., аспирант кафедры автоматизации обработки информации Томского государственного университета систем управления и радиоэлектроники.

Залятин Н.B., доктор технических наук, профессор кафедры автоматизации обработки информации Томского государственного университета систем управления и радиоэлектроники.

Cлирнов Г.В., доктор технических наук, профессор кафедры радиоэлектронных технологий и экологического мониторинга Томского государственного университета систем управления и радиоэлектроники. 


\title{
DISINFECTION AND USAGE OF ANTHROPOGENIC WASTE IN RESOURCE-SAVING CONSTRUCTION TECHNOLOGIES
}

\author{
Vladimir I. Makovkin', \\ assorti2030@yandex.ru \\ Nikolay V. Zamyatin', \\ zamnv@gmail.com \\ Gennady V. Smirnov', \\ smirnov@main.tusur.ru \\ 1 Tomsk State University of Control Systems and Radioelectronics, \\ 40, Lenin Avenue, Tomsk, 634050, Russia.
}

Relevance. The environmental protection problem is one of the most important one in the present time. Multilateral pollution of all geospheres with non-utilized wastes led to a sharp deterioration of ecological systems, to the death of some unique natural complexes, to the reduction and disappearance of populations of certain plant and animal species, to the danger of unpredictable irreversible consequences which can be the result of man's technogenic activity. That is why the utilization of industrial wastes of industries and their use in resource-saving technologies, of course, are very relevant.

The aim of the work is to show the possibility of utilization, control and use of waste products, in particular fluorine-anhydrite in resource-saving construction technologies.

Methods: chemical waste neutralization, disintegration of the obtained raw materials and production of bulk clinker material from it, methods of its control and application in various formulations in construction technologies; laser scanning of the surface of the received bulk mixture; approximation of the surface model using radial basis function neural networks; modeling of a non-linear surface function, by the Kolmogorov theorem, using the superposition of radial basis functions; calculation of the volume of a substance limited by the obtained function using the cubature function by the Gauss-Kronrod method and the Monte Carlo method.

Results. The paper considers the technology of calcium sulfate neutralization and technological scheme of its disintegration and use in drilling fluids and introduces the system for controlling the volume of a disintegrated substance, which is necessary to account its quantity and dosing when used in formulations of various technologies. It is shown that when controlling and estimating the amount of a disintegrated substance, the best way is to approximate the nonlinear function, since it is possible to control the error by the number of partitions of the function or the number of experiments, and also the model of the surface of the substance is taken into account. The usage of the radial basis function neural network is advisable for obtaining a model of the surface of bulk materials in order to improve the accuracy of measuring volumes in silo farms and warehouses of manufacturing enterprises. Methods of modeling surface functions and measuring their volume by quadrature or Monte Carlo methods are the most effective ones. Usage of the Gauss-Kronrod method in this case is preferable.

\section{Key words:}

Industrial waste, resource-saving technologies, silo, level gauge, volume, bulk material, neural network, Radial Basis Function neural network, quadrature, approximating function, interpolating function, Matlab.

\section{REFERENCES}

1. Fedorchuk Yu.M., Zykova N.S., Mikheev V.N. The multipurpose use of industrial wastes in the process of producing anhydrite construction materials. Strategic Technology (IFOST). $7^{\text {th }}$ International Forum. Tomsk, Russia, 2012. 3 p.

2. Anikanova L., Volkova 0., Guseva I., Kurmangalieva A. Research of Gypsum-Containing Raw Materials for Obtaining of Dry Building Mixes. Key Engineering Materials, 2015, vol. 683, pp. $295-300$.

3. Gutt W. Manufacture of cement from industrial byproducts. Chemistry and Industry, 1971, no. 7, pp. 189-197.

4. Garkavi M.S. Anhydrite Floors for Civil Construction. Proc. of the $14^{\text {th }}$ Internationale Baustofftagung «Ibausil». Weimar, Germany, 2000. pp. 865-870.

5. Wang Jinhua, Yang Xinya, Yu Degao. Modification of fluorgypsum as cement retarder. Journal of Wuhan University of Technology-Mater. Sci.Ed., 2007, vol. 22, no. 4, pp. 745-748.

6. Ludvigsen K. Production of gypsum boards from gypsum waste. Cim., betons, platres, chaux, 1998, no. 2, pp. 97-98,

7. Makovkin V.I., Zamyatin N.V. System for monitoring and processing information on the state of the silo farm. Doklady Tomskogo gosudarstvennogo universiteta sistem upravleniya i radioehlektroniki, 2016, no. 19 (4), pp. 95-99. In Rus.
8. Fedorchuk Yu.M., Zamyatin N.V., Smirnov G.V., Rusina 0.N., Sadenova M.A. Prediction of the properties anhydrite construction mixtures based on neural network approach. Journal of Physics: Conference Series, 2017, no. 881, 6 p.

9. Fedorchuk Yu.M. The results of commissioning and technological tests of production of anhydrite unification, obtained from solid waste of hydrofluoric production of the Siberian Chemical Combine. Khimicheskaya promyshlennost, 2004, no. 3, pp. 113-115. In Rus.

10. Fedorchuk Yu.M., Goryukhin K.E., Manankov A.V., Nedavniy 0.I., Erdniev A.I. Malogabaritnoe zdanie iz betonnoy smesi [A small-sized building made of concrete mix]. Patent RF, no. 35540, 2004.

11. Smirnov G.V, Zamyatin N.V. Sposob kontrolja siputschich materjalov $v$ reservuarach [Method of control of parameters of loose materials in tanks]. Patent RF, no. 2661314, 2018.

12. Kolesnikov V.A. Sredstva izmereniya i kontrolya tekhnologicheskikh protsessov na predpriyatiyakh gorno-metallurgicheskogo kompleksa [Instruments of measurement and control of technological processes at the enterprises of the mining and metallurgical complex]. Karaganda, KarGTU Publ., 2012. 107p.

13. Yakimovich E.A., Zamyatin N.V. Modelirovanie beskontaktnogo videourovnemera s ispolzovaniem analiza neyronnykh setey [Simulation of a contactless video level meter using neural network 
analysis]. Nauchnoe izdanie «Informatsionnye tekhnologii v territorialnom upravlenii, promyshlennosti, obrazovanii». Tomsk, 2002. pp. 165-170.

14. Smirnov G.V., Smirnov D.G. Imbibition of windings of electric machines with a magnetodielectric composite with ultradisperse nickel-zinc filler. Doklady Tomskogo gosudarstvennogo universiteta sistem upravleniya i radioehlektroniki, 2016, no. 19 (2), pp. 99-102. In Rus.

15. Rybakov A.G., Lukyanova E.V., Burmistrova I.S., Muratova Yu.F., Fedorova M.V. Methods for measurement of volume of bulk material. Energeticheskie i elektrotekhnicheskie sistemy, 2015, vol. 2, pp. 430-433. In Rus.

16. Kulazhsky A.V. Application of neural networks based on radial basis functions in modeling terrain. Vestnik RGUPS, Zheleznodorozhny put $i$ transportnoe stroitelstvo, 2009, no. 3, vol. 35, pp. 95-100. In Rus.

17. Konovalov N.E., Zonnekh N.N., Melnik I.G., Yakimovich A.K. Obosnovanie matematicheskoy modeli mestnosti dlya proektirovaniya avtomobilnykh dorog [Verification of mathematical model of terrain for designing motor roads]. Nauch. Tr. Giprodornii, 1976, no. 18, $7 \mathrm{p}$.

\section{Information about the authors}

Vladimir I. Makovkin, postgraduate student, Tomsk State University of Control Systems and Radioelectronics.

Nikolay V. Zamyatin, Dr. Sc., professor, Tomsk State University of Control Systems and Radioelectronics.

Gennady V. Smirnov, Dr. Sc., professor, Tomsk State University of Control Systems and Radioelectronics.S
18. Filatova T.V. Primenenie neyronnyh setey dlya approksimatsii dan nykh [Application of neural networks for data approximation]. Tomsk, Tomsk State University Press, 2004. 30 p.

19. Kolmogorov A.N. 0 predstavlenii nepreryvnykh funktsy neskolkikh peremennykh superpozitsiyami nepreryvnykh funktsy menshego chisla peremennykh [On presentation of continuous functions of several variables by superpositions of continuous functions of fewer variables]. Doklady AN USSR, 1956, no. 108, pp. $179-182$.

20. Huang Y., Chen B., Chen G., Xiao H., Khan S.U. Simultaneous detection of liquid level and refractive index with a long-period fiber grating based sensor device. Measurement Science and Technology, 2013, vol. 24 (9), 095303.

21. Khaliq S., James S.W., Tatam R.P. Fiber-optic liquid-level sensor using a long-period grating. Opt. Lett., 2001, vol. 26, pp. 1224-1226.

Received: 15 February 2018. 